\title{
Competências de Comunicação Clínica: Objetivos de Ensino-Aprendizagem para um Currículo Nuclear nas Áreas da Saúde
}

\author{
Clinical Communication Skills: Teaching- \\ Learning Objectives for a Core Curriculum \\ in the Areas of Health
}

\author{
Elizabete Loureirol \\ Afonso Miguel Cavaco ${ }^{I I}$ \\ Maria Amélia Ferreira ${ }^{I}$
}

\section{PALAVRAS-CHAVE \\ - Competências de Comunicação Clínica; \\ - Currículo; \\ - Ciências da Saúde; \\ - Educação Médica.}

\section{KEYWORDS}

- Clinical Communication Skills;

- Curriculum;

- Health Sciences;

- Medical Education.
Enviado em: 05/12/2013

Reencaminhado em: 10/09/2014

Aprovado em: 08/08/2015 


\section{INTRODUÇÃO}

A importância das competências e aptidões para a comunicação clínica (CCC) nas profissões da saúde tem sido reconhecida em todo o mundo ${ }^{1}$. Estas competências na entrevista clínica, no exame físico e na coleta de dados objetivos são procedimentos vitais e nucleares que melhoram significativamente a prestação de cuidados de saúde e revelam-se instrumentos poderosos de diagnóstico e tratamento ${ }^{2}$.

Não obstante este reconhecimento, estão bem documentados problemas relacionados com o processo de ensino-aprendizagem das CCC e que contribuem para situações de erro diagnóstico, falta de adesão à terapêutica, baixa satisfação dos pacientes e profissionais de saúde, problemas médico-legais, ou seja, menor efetividade e aumento dos custos para os sistemas de saúde ${ }^{3,4}$.

Considerando que a comunicação clínica é um conjunto de competências adquiridas ${ }^{5}$, vários estudos indicam que a formação específica em competências de comunicação leva a melhorias significativas na relação profissional de saúde-paciente, não só a curto, mas também a longo prazo, e a melhores resultados em indicadores de saúde ${ }^{6,7}$. As consequências da aptidão para bem comunicar estão vertidas, por exemplo, no desenvolvimento de modelos centrados no paciente e na relação terapêutica, que contrariam o modelo biomédico muitas vezes dominante nos cuidados de saúde atuais ${ }^{3,8}$. Se a comunicação em saúde for abordada numa perspectiva interacional e de parceria, em que os profissionais de saúde e os pacientes partilham as tomadas de decisão clínica ${ }^{9}$, contribui-se no sentido de intervenções mais eficazes e na prestação de cuidados de saúde de elevada qualidade.

Todavia, observam-se dificuldades na implementação de programas de formação em CCC. Entre as questões recorrentemente levantadas por investigadores ${ }^{10}$ encontram-se a melhor forma de ensino para promover a aprendizagem dos vários aspetos da relação profissional de saúde-paciente, quais os componentes mais relevantes dessa relação para a prestação otimizada dos cuidados, e quais as perceções de estudantes e docentes em face do processo de ensino-aprendizagem. A investigação defende que estes programas apresentam efeitos positivos, mas as linhas de orientação e temáticas dos conteúdos devem ser claramente predefinidas ${ }^{11,12}$, ainda antes dos métodos pedagógicos e da avaliação da aquisição de competências.

Tem-se ainda verificado um défice no processo de observação e avaliação estruturado das competências por parte dos docentes clínicos 3 . Porém, o ensino das competências de comunicação "requer um grande número de facilitadores e torna-se primordial que os docentes tenham competências clí- nicas eficientes, sejam capazes de observar, avaliar e fornecer feedback aos estudantes" ${ }^{\prime 3}$. Desta forma, é necessário desenvolver um referencial de trabalho não só para as bases dos currículos em CCC, mas também para o ensino sistemático da comunicação, fornecendo treino específico aos docentes clínicos, colocando maior ênfase no desenvolvimento de competências pedagógicas e clínicas. A revisão da literatura relata alguns programas de formação pedagógica destinados a promover o ensino de conteúdos específicos, tais como as competências de comunicação ${ }^{13}$.

As competências de comunicação com os pacientes, seus familiares/cuidadores e equipes de saúde são transversais nas profissões em que existem cuidados, e por isso a comunicação clínica efetiva deve ser um objetivo nuclear da educação de todos os profissionais de saúde. Deste modo, considera-se pertinente a definição de competências de comunicação clínica partilhadas pelos diferentes profissionais, as quais deveriam ser incluídas nos currículos de formação pré-graduada desses profissionais ${ }^{14}$. Não obstante, parece não existir evidência na literatura de consensos sobre comunicação clínica em áreas não médicas. Muitas organizações médicas têm descrito a importância da integração das CCC como parte fundamental do currículo médico ${ }^{15-21}$, mas são escassos os trabalhos sistemáticos sobre o treino em comunicação clínica para outros grupos profissionais $22-25$.

Por outro lado, Razack et al. ${ }^{26}$ destacam que as aptidões nucleares de comunicação clínica são muitas vezes consideradas competências soft, sendo frequentemente ensinadas de forma implícita, por meio da sua observação mais ou menos estruturada, e assim fazendo parte de um currículo oculto. Contrariando essa perspetiva, surgiu, em 2000, a Associação Europeia para a Comunicação em Saúde $(\mathrm{EACH})^{27}$, fundada por investigadores, clínicos e profissionais associados à educação médica de diferentes países europeus. Seu propósito, tal como na organização norte-americana congênere (American Academy on Communication in Health Care - ACCH), é estabelecer uma rede multidisciplinar de peritos ativamente envolvidos em investigação e ensino da comunicação clínica, de modo a contribuir para a melhoria contínua da qualidade da prestação de cuidados de saúde. Integrado na $\mathrm{EACH}$, o Comitê de Ensino da EACH (tEACH) ${ }^{28}$, criado em 2008, tem o objetivo de providenciar apoio, recursos e estimular o trabalho em rede de docentes e formadores de CCC. O tEach é atualmente constituído por cinco subgrupos - Core Curriculum, Teaching Tools, Assessment, Web-Based Networking, Train the Trainers -, cujos objetivos são contribuir para a missão principal do comitê. O subgrupo Core Curriculum aceitou o desafio de desenvolver e alcançar um consenso para um currículo nuclear em 
comunicação clínica, transversal ao ensino pré-graduado de todas as profissões de saúde na Europa.

Desta forma, em 2011, através de uma abordagem acadêmica sistemática, utilizando um processo Delphi-like $e^{29-31}$, foi construído o Health Professions Core Communication Curriculum - HPCCC ${ }^{32}$, com a colaboração de 121 especialistas em comunicação clínica de 15 áreas profissionais ligadas à saúde, tais como Medicina, Enfermagem, Farmácia, Psicologia, Odontologia, Fisioterapia e Fonoaudiologia, de 16 países europeus.

O HPCCC identifica 61 objetivos de ensino-aprendizagem a serem alcançados nos ciclos de formação pré-graduada de cursos das ciências da saúde, reforçando os objetivos do processo de Bolonha. Sua aplicabilidade prática está na sua potencialidade para servir como uma referência para instituições e profissões das áreas da saúde quanto ao planejamento e implementação ou reestruturação dos conteúdos curriculares de ensino e avaliação da comunicação clínica. Sugere-se utilizar este instrumento como um referencial flexível, podendo ser adaptado como um todo ou parcialmente, de acordo com as necessidades e especificidades institucionais e/ou dos grupos profissionais.

O subgrupo do tEACH responsável pela construção do HPCCC tem agora o desafio, em conjunto com os outros subgrupos, de: (i) complementar os objetivos pedagógicos com ferramentas de ensino e avaliação apropriados; (ii) fornecer apoio a programas de treino de formadores clínicos e sistemas de avaliação; (iii) explorar a importância dos objetivos do HPCCC na perspetiva dos estudantes e dos pacientes; (iv) avaliar os objetivos dentro das diferentes áreas de cuidados e suas culturas.

Em Portugal, os cursos das áreas da saúde parecem não ter instituído um processo formal, específico e integrado de ensino e avaliação das CCC. Muito recentemente e na tentativa de compensar essa deficiência, têm-se criado cursos de atualização e de pós-graduação ${ }^{33-35}$ e cursos de formação pedagógica ${ }^{36}$. Contudo, não sendo de caráter obrigatório, têm alcance limitado e possivelmente insuficiente. No Brasil, apesar de também se verificar uma carência de literatura específica sobre o processo de ensino-aprendizagem das CCC, há estu$\operatorname{dos}^{37-40}$ que salientam a necessidade de rever este tema para a sua incorporação de forma mais incisiva (e não subliminar) nos cursos de formação em saúde (por exemplo, em diretrizes curriculares nacionais dos cursos de Medicina e Enfermagem).

Para que as competências de comunicação clínica possam ser continuamente melhoradas e sustentáveis, é necessário que se reforce a implementação de um suporte à investigação e avaliação, sequenciais aos programas de treino destas competências. Neste contexto, sugere-se a criação de redes nacionais de profissionais das áreas das ciências da saúde de modo a: (i) desenvolver um consenso sobre os objetivos de ensino-aprendizagem para um currículo nuclear em competências de comunicação clínica, com base na tradução portuguesa já efetuada do HPCCC; (ii) otimizar ferramentas de ensino-aprendizagem e de avaliação sistemáticas e eficazes; (iii) efetuar estudos de investigação sobre o desenvolvimento dos programas, seu impacto e resultados na aprendizagem, intra e interprofissões de saúde. Este é o momento para alavancar as várias iniciativas que estão no terreno para a comunicação em saúde em língua portuguesa.

\section{AGRADECIMENTOS}

Agradecemos à Fundação para a Ciência e Tecnologia o apoio financeiro por meio do projeto COSTProMed - PTDC/SAU-SAP/1112908/2009, ao Comitê de Ensino da EACH (tEACH) e aos restantes membros do subgrupo Core Curriculum.

\section{REFERÊNCIAS}

1. Parrot R. Emphasizing "communication" in health communication. J Commun. 2004;54:751-87

2. Rotthoff T, Baehring T, David D, Bartnick C, Linde F, Willers $\mathrm{R}$, Schafer $\mathrm{R}$, Scherbaum $\mathrm{W}$. The value of training in communication skills for continuing medical education. PatientEducCouns. 2010;84:170-75.

3. Kurtz S, Silverman J, Draper J. Teaching and Learning Communication Skills in Medicine. Oxford: Radcliff Publishing 2005;13-20.

4. Maguire P, Pitceathly C. Key communication skills and how to acquire them. BMJ. 2000;325:697-700.

5. Kurtz S, Laidlaw T, Makoul G, Schnabl G. Medical Education Iniciatives in Communication Skills. Cancer Prev Control. 1999; 3:37-45.

6. Yedida M, Gillespie C, Kachur E, Schwartz M, Ockene J, Chepaitis A, Snyder C, Lazare A, Lipkin M. Effectofcommunications training on medical student performance. JAMA 2007; 290(9):1157-1165.

7. Laidlaw T, MacLeod H, Kaufman D, Langille D, Sargeant J. Implementing a communication skills programme in medical school: needs, assessment and programme change. Med Educ. 2002; 36:115-124.

8. Brown J. How clinical communication has become a core part of the medical education in the UK. Med Educ. 2008;42:271-8

9. Charles C, Whelan T, Gafni A. What do we mean by partnership in making decisions about treatment? BMJ. 2007;319:780-2.

10. Wright K, Bylund C, Ware J, Parker P, Query J, Baile W. Medical Student Attitudes towards communication skills 
training and knowledge of appropriate provider-patient communication: a comparison of first-year and fourth-year medical students. Med Educ. 2006;7:3.

11. Veldhuijzen W, Ram P, Weijden T, Wassink M, Vleuten, C. Much variety and little evidence: a description of guidelines for doctor-patient communication. Med Educ. 2007;41(2):138-145.

12. Spencer J, Silverman J. Education for communication: much already known, so much more to understand. Med Educ. 2001; 35(3):188-90.

13. Steinert Y, Mann K. Faculty development: principles and practices. JVME. 2006; 33(3).

14. Razack S, Meterissian S, Morin L, Steinert Y, Tabatabai D, MacLellan A. Coming of age as communicators: differences in the implementation of common communications skills training in four residency programmes. Med Educ. 2007;41:441-9.

15. Makoul G, Schofield T. Communication teaching and assessment in medical education: an international consensus statement. Patient Educ Couns. 1999;37:191-5.

16. Simpson M, Buckman R, Stewart M, Maguire P, Lipkin M, Novack D, Till J. Doctor-patient communication: the Toronto consensus statement. BMJ. 1991; 30; 303:1385-7.

17. Makoul G. Essential elements of communication in medical encounters: the Kalamazoo consensus statement. Acad Med. 2001; 76: 390-3.

18. Von Fragstein M, Silverman J, Cushing A, Quilligan S, Salisbury H, Wiskin C. UK Council for Clinical Communication Skills Teaching in Undergraduate Medical Education. UK consensus statement on the content of communication curricula in undergraduate medical education. Med Educ. 2008;42(11):1100-7.

19. Kiessling C, Dieterich A, Fabry G, Holzer H, Langewitz W, Mullinghaus M, Pruskil S, Scheffer S Schubert S. Communication and social competency in medical education in German speaking countries: The Basel Consensus statement. Resultsof a Delphi Study. Patient Educ Couns. 2010; 81:259-66

20. Frank JR, ed. The CanMEDS physician competency framework. Better standards. Better physicians. Better care. 2005 Ottawa: The Royal College of Physicians and Surgeons of Canada.

21. Makoul G, Schofield T. Communication teaching and assessment in medical education: an international consensus statement. Netherlands Institute of Primary Health Care. Patient Educ Couns. 1999; 37:191-5.

22. Fellowes D, Wilkinson S, Moore P. Communication skills training for health care professionals working with cancer patients, their families and/or carers. The Cochrane Database of Systematic Reviews. 2004; 2.

23. Krijver IPM, Kerkstra A, Bensing JM, Van de Wiel HBM. Nurse-patient communication in cancer care: a review of the literature. Cancer Nurs. 2000; 23: 20-31.

24. Ajjawi R, Higgs J. Core components of communication of clinical reasoning: a qualitative study with experienced Australian physiotherapists. Adv Health Sci Educ Theory Pract. 2012;17:107-19.

25. Holt J, Coates C, Cotterill D, Eastburn S, Laxton J, Mistry $\mathrm{H}$, Young $\mathrm{C}$. Identifying common competences in health and social care: an example of multi-institutional and inter-professional working. Nurse Educ Today. 2010;30:264-70.

26. Razack S, Meterissian S, Morin L, Steinert Y, Tabatabai D, MacLellan A. Coming of age as communicators: differences in the implementation of common communications skills training in four residency programmes. Med Educ. 2007;41:441-9.

27. European Association for Communication in Healthcare. Disponível em: http:/ / www.each.eu/. Acesso em: 10 jan. 2016.

28. European Association for Communication in Healthcare. tEACH. Disponível em: http://www.each.eu/teaching/. Acesso em: 10 jan. 2016.

29. Brown. Delphi Process: A Methodology Used for the Elicitation of Opinions of Experts. 1968. Disponível em: http:/ / www.rand.org/pubs/papers/2006/P3925.pdf

30. De Villiers MR, de Villiers PJT, Kent AP. The Delphi technique in health sciences education research. Med Teach 2005; 27:639-43.

31. Fink A, Kosecoff J, Chassin M, Brook RH. Consensus methods: characteristics and guidelines for use. Am J Public Health 1984, 74: 979-83

32. Bachmann C, Abramovitch H, Barbu CG, Cavaco AM, Elorza RD, Haak R, Loureiro E, Ratajska A, Silverman J, Winterburn S, Rosenbaum M. A European consensus on learning objectives for a core communication curriculum in health care professions. Patient EducCouns. 2013;93(1):18-26.

33. Faculdade de Medicina da Universidade do Porto. Competências de Comunicação Clínica. Disponível em: https://sigarra.up.pt/fmup/pt/cur_geral.cur_view?pv_ ano_lectivo $=2012 \&$ pv_origem $=C U R \& p v \_t i p o \_c u r \_$ sigla=E\&pv_curso_id=1198. Acesso em: 11 nov. 2016

34. Carvalho IP, Pais VG, Almeida SS, Ribeiro-Silva R, Figueiredo-Braga $\mathrm{M}$, Teles A, Castro-Vale I, Mota-Cardoso R. Learning clinical communication skills: Outcomes of a program for professional practitioners. Patient Educ Couns. 2011; 84(1):84-89. 
35. Faculdade de Medicina da Universidade do Porto. Formação em Comunicação Clínica para Médicos Internos. Disponível em: https://sigarra.up.pt/fmup/pt/cur_geral.cur_ view?pv_ano_lectivo $=2012 \& p v \_o r i g e m=C U R \& p v \_t i p o \_$ cur_sigla=A\&pv_curso_id=1124. Acesso em:11 nov. 2016.

36. Centro de Educação Médica. Formação contínua pedagógica. Disponível em: https://sigarra.up.pt/fmup/ pt /cur_geral.cur_view?pv_ano_lectivo $=2015 \&$ pv origem $=C A N D \& p v \_t i p o \_c u r \_s i g l a=C F C \& p v \_c u r s o \_$ id=2221 Acesso em: 11 nov. 2016

37. Silva C, Rodrigues C, Lima J, Jucá N, Augusto K, Lino C, Carvalho A, Andrade F, Rodrigues J, Caprara A. Relação médico-paciente em oncologia: medos, angústias e habilidades comunicacionais de médicos na cidade de Fortaleza (CE). Ciência e Saúde Coletiva. 2011; 16(Supl.1):1457-1465.

38. Ribeiro M, Krupat E, Amaral C. Brazilian medical students' attitudes towards patient-centered care. Medical Teacher. 29:e204-e208

39. Rossi P, Batista N. O ensino da comunicação na graduação em Medicina - uma abordagem. Interface Comunic Saúde Educ. 2006;9(18):93-102.

40. Spagnudo R, Pereira R. Práticas de saúde em Enfermagem e Comunicação: um estudo de revisão da literatura. Ciência e saúde coletiva 2007; 12(6).

\section{CONTRIBUIÇÃO DOS AUTORES}

ELe AMC fazem parte da comité de ensino (tEACH) do EACH e participaram ativamente na elaboração do HPCCC. MAF faz parte da comité de investigação (rEACH) do EACH e tem viabilizado a aplicação do HPCCC no processo de reforma curricular da Faculdade de Medicina da Universidade do Porto. EL, AMC \& MAF contribuíram para a escrita e aprovação da versão final deste ensaio.

\section{CONFLITO DE INTERESSES}

Os autores declaram não haver conflito de interesses.

\section{ENDEREÇO PARA CORRESPONDÊNCIA}

Afonso Miguel Cavaco

Departamento de Educação e Simulação Médica/ Faculdade de Medicina da Universidade do Porto

Alameda Professor Hernâni Monteiro

CEP 4200-319 - Porto - Portugal

Email: emfl@med.up.pt 